\title{
Graphene opens up to new applications
}

\author{
Effective separation membranes could be created by etching nanometre-sized pores in \\ two-dimensional materials.
}

The explosion of research interest in twodimensional materials such as graphene and molybdenum disulphide has, to a large extent, been dominated by their physics, and in turn the exploitation of their electronic and optical properties. Researchers have, of course, also explored the chemical and mechanical properties of these materials - and sought applications that principally utilize these attributes but the results have, arguably, received less attention. One intriguing line of research in this regard is the use of graphene as a nanoporous separation membrane. Here, through a combination of sophisticated fabrication and characterization techniques, unique membranes could be developed for use in critical applications such as gas separation, water purification, and desalination.

Graphene is an attractive material for the development of membranes due to its atomic thickness, mechanical strength and chemical stability. Pristine sheets of graphene are thought to be impermeable to all atoms and molecules. However, by forming nanometre-sized pores in the material, it can potentially act as a filter, allowing molecules smaller than the pores to pass through while excluding larger species. A number of theoretical studies have suggested that the selectivity and permeability of such membranes could be vastly superior to the polymerbased filtration membranes that are typically used today ${ }^{1-3}$. And in the past few years, experimental demonstrations of the potential of nanoporous graphene membranes have begun to emerge ${ }^{4-9}$.

It has, for example, been reported that oxidative etching can be used to create pores in single and bilayer graphene, and the resulting membranes used to selectively transport gases ${ }^{4}$. Alternatively, layered graphene and graphene oxide membranes have been shown to separate carbon dioxide from nitrogen, suggesting that the approach might be of value in carbon capture applications ${ }^{5}$. Similarly, membranes made from two or three layers of graphene oxide can separate hydrogen from carbon dioxide and nitrogen ${ }^{6}$.

A key challenge in the development of these membranes is the fabrication of pores with precise dimensions, and last year it was

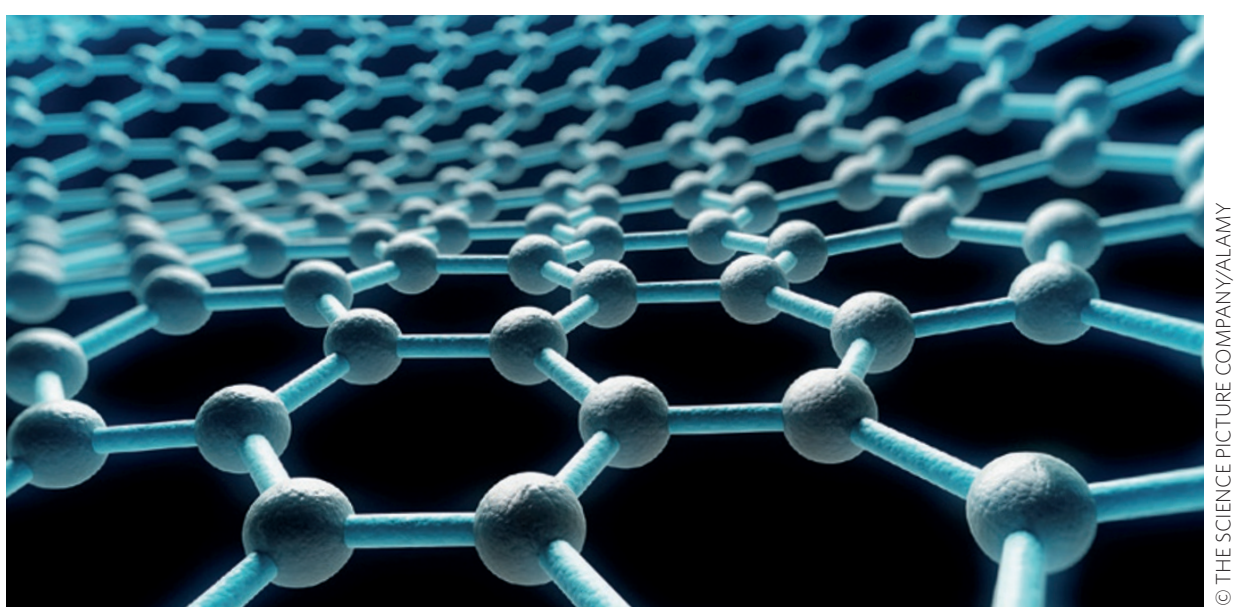

reported that subnanometre pores can be formed over macroscopic areas of graphene through a combination of ion bombardment and chemical etching ${ }^{7}$. With this approach, it was possible to create membranes that can selectively transport ions in solution. Furthermore, it has been shown that a focused ion beam can drill pores with controlled diameters in double-layer graphene ${ }^{8}$. The resulting membranes exhibit gas, liquid, and water vapour permeances that are orders-of-magnitude greater than current state-of-the-art membranes.

Reporting in this issue of Nature Nanotechnology, Ivan Vlassiouk, Shannon Mahurin and colleagues have now shown that a single layer of nanoporous graphene can be used to desalinate water ${ }^{10}$. The pores are created in the graphene layer by exposing the material to short bursts of oxygen plasma, a process that allows holes of precise dimensions to be controllably etched in the layer. By finding just the right plasma conditions - and with the help of aberration-corrected scanning transmission electron microscopy to characterize pores that have sizes of only $0.5-1 \mathrm{~nm}-$ membranes can be fabricated that exhibit a salt rejection of nearly $100 \%$, as well as high water fluxes.

As Dong-Yeun Koh and Ryan Lively note in an accompanying News \& Views article ${ }^{11}$, there are many challenges to be addressed before such membranes could be of practical use, including issues related to mechanical stability and membrane fouling. However, these proof-of-concept experiments are an encouraging illustration of the potential of atomically thick membranes.

Although pore size is critical for desalination, pores might not be required at all for other applications of graphene membranes. Recently, it has been reported that protons can, in fact, be transported through pristine monolayers of graphene ${ }^{9}$. (Other two-dimensional materials were also examined, and it was found that protons can pass through monolayers of hexagonal boron nitride, but not through monolayers of molybdenum disulphide.) The result suggests that graphene might also, therefore, be of use in the development of proton exchange membranes for fuel cells.

Whatever the intended application, graphene membranes provide a fascinating system in which molecular transport can be studied and controlled at the ultimate limit. And through such fundamental studies, practical devices, which might, for example, help to address the increasing global demand for fresh water, could be realized. $\square$

References

1. Sint, K., Wang, B. \& Kral, P. J. Am. Chem. Soc. 130, 16448-16449 (2008).

2. Jiang, D.-e., Cooper, V. R. \& Dai, S. Nano Lett. 9, 4019-4024 (2009).

3. Cohen-Tanugi, D. \& Grossman, J. C. Nano Lett. 12, 3602-3608 (2012)

4. Koenig, S. P., Wang, L., Pellegrino, J. \& Bunch, J. S. Nature Nanotech. 7, 728-732 (2012).

5. Kim, H. W. et al. Science 342, 91-95 (2013).

6. Li, H. et al. Science 342, 95-98 (2013).

7. O'Hern, S. C. et al. Nano Lett. 14, 1234-1241 (2014).

8. Celebi, K. et al. Science 344, 289-292 (2014).

9. Hu, S. et al. Nature 516, 227-230 (2014).

10. Surwade, S. P. et al. Nature Nanotech. 10, 459-464 (2015).

11. Koh, D.-Y. \& Lively, R. P. Nature Nanotech. 10, 385-386 (2015). 